Archivum, LXX (I), 2020, pp. 65-79

\title{
El concepto de verdad en Alain Badiou: de la edad de los poetas a la edad de las matemáticas*
}

\author{
Mauricio Cheguhem Riani \\ UNIVERSIDAD DE SALAMANCA \\ mauriche8@gmail.com
}

Recibido:09/07/2019

Aceptado: 04/10/2019

\section{RESUMEN:}

El presente trabajo pretende abordar el concepto de verdad en Alain Badiou a partir de dos esferas reconocibles del conocimiento filosófico: por un lado, el espacio central que ocupa la poesía desde Nietzsche hasta Heidegger en la disposición filosófica; y, por otro, la necesidad de transportarlo al campo de la lógica matemática. De esta manera, podemos analizar la propuesta de Badiou como un pensamiento superador de "las dos culturas" $y$, a su vez, como un intento por unificar aquello que había sido clausurado bajo el nombre de "la guerra de las ciencias". Por ello, en este trabajo no son ajenas las estéticas rupturistas de finales de siglo xIx y principios del xx, así como tampoco las

* Candidato a doctor por la Universidad de Salamanca en Español: estudios avanzados en lengua y literatura. Este artículo ha sido realizado en el marco de las actividades financiadas por el Proyecto de I+D de Excelencia Inscripciones literarias de la ciencia: cognición, epistemología y epistemocrítica (ILICIA) del Ministerio de Economía y Competitividad. Ref. FF12017-83932-P. 
influencias de matemáticos como Brouwer, Cantor y Gödel. De este modo, para que la filosofía salga del ostracismo metafísico del siglo pasado, y como salto fundacional sobre el enunciado fin de la filosofía, es imperativo un retorno clásico que vuelva a poner en diálogo poema y matema: literatura y matemáticas.

PALABRAS CLAVE: Alain Badiou, Verdad filosófica, Filosofía de la Ciencia, Estética, Literatura y Ciencia.

The concept of truth in Alain Badiou: from the age of poets to the age of mathematics

\begin{abstract}
:
The present work aims to address the concept of truth in Alain Badiou from two recognizable spheres of philosophical knowledge; on the one hand, the central space occupied by poetry from Nietzsche to Heidegger in the philosophical disposition, and on the other, the need to transport it to the field of mathematical logic. In this way, we can analyze Badiou's proposal as a thought that overcomes the "two cultures" and, in turn, as an attempt to unify that which had been closed under the name of "the war of the sciences". For this reason, in this work the rupturistic aesthetics of the end of the 19th and early 20th centuries, as well as the influences of mathematicians such as Cantor and Gödel are not strange. In turn, for philosophy to emerge from the metaphysical ostracism of the 20th century, and as a foundational leap over the enunciated end of philosophy, a classic return is imperative that brings poetry and mathematics back into dialogue: literature and mathematics.
\end{abstract}

KEY WORDS: Alain Badiou, Philosophical Truth, Philosophy of Science, Aesthetics, Literature and Science.

\title{
1. Del concepto de verdad
}

Toda definición de la filosofía debe distinguirla de la sofística. Esta tesis obliga en suma a abordar la definición de la filosofía mediante el concepto de verdad. Puesto que lo que el sofista, antiguo o moderno, pretende imponer es precisamente que no hay verdad, que el concepto de verdad es inútil e incierto, ya que no hay sino convenciones, reglas, géneros del discurso o juegos de lenguaje. 
Alain Badiou demuestra especial interés por los espacios de fricción entre las distintintas esferas del conocimiento que circulan en su filosofía desde una perspectiva reaccionaria y atenta. Preocupado por esta ruptura, apunta al período romántico como el verdadero responsable del desencuentro entre ciencia y humanidades. En el mismo sentido, C. P. Snow cifra en Las dos cultuas un divorcio histórico que se inicia en los albores del s. XIX $\mathrm{y}$ estalla en pleno siglo $\mathrm{xx}$ con la tan anunciada "guerra de las ciencias". ${ }^{1}$

En el campo de la filosofía, Badiou no vacila en denunciar que el pensamiento decimonónico comienza con un lento pero firme paso hacia la anulación del poder de las matemáticas sobre el razonamiento metafísico. En efecto, el siglo xIx "va a organizar la convicción de que la filosofía puede y debe desplegar un pensamiento que no interiorice en ningún momento a la matemática como condición de su despliegue" (Badiou, 2012, 153). De esta manera, lo que las humanidades, y la filosofía en particular, relativizan en torno a la matemática no es tan sólo su condición metafísica, sino fundamentalmente su sentido de verdad.

En el intento por establecer un diálogo transdisciplinario, Badiou propone un nuevo enfoque que permita un diálogo entre humanidades y ciencias, precisamentente entre poesía y matemática. Más aún, el francés advierte que poder volver a unir ciencia y literatura es la operación necesaria para quien quiera superar la estela del pensamiento romántico y recuperar definitivamente el discurso metafísico. Sin embargo, este trayecto lo transporta por caminos sinuosos como lo son la literatura, la política, la ciencia, el psicoanálisis y la estética. Es importante, en este sentido, advertir las dificultades que presenta su escritura como espacio plural e inasible de ciertas áreas específicas que

$1 \mathrm{Al}$ respecto, el físico y escritor inglés asegura: “El surgimiento de la angustia ante la posibilidad de que se produjera una fisura semejante en los tipos de conocimiento, perjudicial tanto para la cultura individual como para el bienestar social, puede fecharse en el período romántico, entre finales del siglo xvıI y principios del siglo XIX" (Snow, 2000, 9). 
atraviesan los núcleos de su obra, tales como la ontología, la matemática y la poesía.

Al mismo tiempo, Badiou no participa de la corriente posmetafísica, poshistórica, posmoderna, en la medida en que no cree que la disciplina se encuentre en una suerte de final de los tiempos. En este sentido, propone retomar el lenguaje filosófico de la modernidad que va desde Descartes hasta Nietzsche: el anudamiento entre ser, verdad y sujeto (Badiou, 2007, 11). ${ }^{2}$ Lo novedoso de su propuesta recae precisamente en la defensa cerrada del concepto de verdad en las postrimerías del siglo. El paso que conmina el francés es de orden platónico: la verdad debe ser la única y real búsqueda de cualquier filosofía posible. Esta premisa obliga a distinguir la filosofía de la sofística, sea esta antigüa o moderna (Badiou, 2012, 55).

Para ello, en primer lugar, se debe afirmar que la filosofía hace un ejercicio de captura de la verdad a partir de diferentes medios o 'condiciones' que son necesariamente extrafilosóficas. En Manifiesto por la filosofía escribe que las 'condiciones' son transversales a cualquier metafísica: "se trata de procedimientos uniformes, reconocibles a distancia, y cuya relación con el pensamiento es relativamente invariable. El nombre de esta invariación es evidente: se trata del nombre 'verdad"' (Badiou, 2007, 13). Badiou menciona cuatro posibilidades que por su entidad interpelan directamente el campo de la filosofía: éstas son la ciencia, la política, el arte y el amor (Badiou, 2012, 71).

Este acto inaugural, sin embargo, no procura erigirse en función de la noción de verdad metafísica, lógica u ontológica de antaño. La arquitectura descansa en un concepto más inofensivo: nos referimos aquí al concepto de 'acontecimiento'. Cabe señalar que

2 La observación de Hersch sobre el cambio anunciado por Badiou en la filosofía resulta esclarecedor para estos propósitos: “Quisiera introducir aquí una observación. Los filósofos de aquella época, Descartes, Spinoza y Leibniz presentan un rasgo común. Para ellos 'es natural' que exista el ser y no es natural, en absoluto, que pueda existir primero la nada. En nuestra época, en cambio, Heidegger invierte esta relación y plantea la siguiente cuestión: ‘Por qué existe algo y no la nada?"' (Hersch, 2010, 141). 
entendemos por acontecimiento aquello que no solamente aparece en el horizonte del sentido, sino que redefine los contornos de la propia disciplina (ya sea científica, artística, política o amorosa). ${ }^{3}$ Para Badiou, como muchos otros filósfos posheideggerianos, el acontecimiento ocupa un rol capital: es la forma violenta de un 'de-velamiento'. García Ponzo ilumina en este sentido: “las verdades son, como puede entreverse en la extraña construcción de esta frase, efectos de una ruptura" (García Ponzo, 2011, 25).

De esta manera, Badiou enlaza los discursos matemáticos y poéticos bajo un régimen disruptivo y violento. Empero, el concepto de verdad no está asociado a un instante (no es, en efecto, un de-velamiento místico o epifánico) sino más bien al recorrido temporal de su sentido. Por ejemplo, el filósofo señala que "poema y matema están, si se los examina desde la filosofía, tanto el uno como el otro, inscritos en la forma general de un procedimiento de verdad" (Badiou, 2009, 67). Pero para ello, es necesario la superación de aquello que el filósofo entiende como la "edad de los poetas".

\section{De la edad de los poetas}

El arte es lo supremo para el filósofo, justo porque él le abre lo más sagrado, el lugar donde en eterna y originaria unión arde, por así decirlo, en una sola llama lo que en la naturaleza y la historia está separado, y lo que, en la vida y la acción, así como en el pensamiento, tiene que escapársenos eternamente.

Schelling (en Habermas, 2012, 105)

"En filosofía hay que tener adversarios" asegura Alain Badiou. ${ }^{4}$ ¿Pero a qué se refiere exactamente? Su entrada en escena se fundamenta sobre el giro efectuado por tres figuras esenciales

3 En el mismo sentido se expresa Abraham Rubín: acontecimiento es "aquello que no ocurre simplemente dentro del horizonte dado de lo que parece ser 'posible', sino aquello que redefine los contornos mismos de lo que es" (Rubín, 2016, 283).

4 Esta polémica ha sido extraída de una entrevista publicada por el diario $\mathrm{La}$ Nación de Argentina. (Santiago, 2012). 
de la posmodernidad: Nietzsche, Wittgenstein y Heidegger. La crítica atiende especialmente al vaciamiento de los conceptos de verdad, ser y en menor medida del sujeto. Lo que evidencia el pensamiento posmoderno es el modo en el que el lenguaje comienza a monopolizar la especulación filosófica. Tanto el giro lingüístico realizado por Wittgenstein como la ontologización del poema en Heidegger indican este proceso. Desde el punto de vista del francés, la filosofía abandona paulatinamente el lenguaje científico y específicamente el matemático. Ese espacio ontológico, en cambio, lo ha ocupado con éxito el pensamiento poético.

Porque hubo realmente, en el tiempo de la desherencia suturada de los filósofos, una edad de los poetas. Hubo un tiempo, entre Hölderlin y Paul Celan, en el que el sentido tembloroso de lo que era el tiempo mismo, el modo de acceso más abierto a la cuestión del ser [...] fueron descubiertos y detentados por el poema.

(Badiou, 2007, 44)

Por esta razón señala a Nietzsche como "el Príncipe (entendiendo Príncipe como 'principio') de la sofística moderna" (Badiou, 2012, 69). De hecho, cuando Nietzsche sentenció el fin de aquello que él llamaba "la enfermedad Platón", no sólo dectreta el fin de la metafísica, sino que trasladó su función al campo de la poesía. En definitiva, lo que 'es' es el poema. Por tanto, el arte configura (ya hacia principios del romanticismo, pero radicalizado en la hermenéutica alemana) la matriz ontológica de la filosofía. En el mismo sentido, Habermas había advertido sobre el papel activo de la filosofía por integrar el arte y contrarrestar de esta manera los desgarramientos de la modernidad (Habermas, 2012, 116).

De esta manera, la filosofía escudriña en la forma poética su sentido de verdad. Pero como para Badiou la verdad se instala a partir de un acontecimiento, cabe apuntar los hiatos, las rupturas que la alta poesía - fundamentalmente franco-germáni- 
ca - ha fraguado en el pensamiento contemporáneo. Por ello, Mallarmé, Rimbaud, Paul Valéry, Trakl (entre otros) no sólo constituyen el panteón de su pensamiento, sino que también revelan el temperamento trasgresor de sus influencias. En definitiva, lo que Badiou busca en la poesía es su capacidad por desautomatizar, romper y transgredir la lógica del lenguaje como un acontecimiento no tan sólo lingüístico sino fundamentalmente ontológico.

Por ello, lo que le atrae de Mallarmé no es su capacidad por no nombrar el objeto, sino para hacer una presentación de la presentación. Es decir: una operación exegética de la materia al servicio de la lengua. A este movimiento de la conciencia poética Badiou denomina "transposición", que se refiere a la capacidad de abstracción donde lo que prevalece no es un objeto sino fundamentalmente una Idea. Esta operación que menciona el francés es la forma seminal de un acontecimiento. Así, la poesía inaugura una verdad porque se proyecta en ella un quiebre en la unidad del sentido: lo que Mallarmé entiende por "vuelo tácito de abstracciones" (Badiou, 2009, 77). Esta ruptura que 'de-vela' la poesía, tanto al nivel del objeto como del sujeto poético, se manifiesta de modo corelacional entre ambas fuerzas.

Luego, Badiou evoca a Heidegger como la conclusión final de este proceso. Un programa, posmetafísico por excelencia, que atiende al ser en el entramado artístico-poético. ${ }^{5}$ Heidegger es, a efectos, el paso consagratorio de la "edad de los poetas". Sin embargo, Badiou no lo acusa por entronar el poema - en efecto esta edad va desde Hölderlin a Paul Celan - sino por vaciar la propuesta filosófica de ciencia y matemática: "Por eso la única crítica fundamental a Heidegger sería la siguiente: la edad de los

5 Cabe señalar que el cambio paradigmático en Heidegger se efectúa sobre el concepto de verdad. Muñoz Martínez apunta: "En consonancia con su modo de pensar y con su interpretación de la filosofía y la cultura de occidente, Heidegger critica el concepto de verdad como 'adecuación', desarrollado desde la filosofía griega y tematizado explícitamente por Tomás de Aquino, e interpreta la verdad como 'des-ocultación'”' $(2006,17)$. 
poetas concluyó, es necesario de-suturar también la filosofía de su condición poética" (Badiou, 2007, 47).

Por consiguiente, si por un lado Badiou entiende que el poema es una condición de la filosofía, y en este sentido espacio de verdades, asume por otro que no es la poesía la única casa del ser. En efecto, lo que advierte el autor es que la filosofía no debe permanecer detenida en la condición del poema. El despliegue misterioso (al decir de Mallarmé) del pensamiento poético pone en altercado la propia capacidad de la filosofía para capturar en los versos su sentido de verdad; se aproxima entonces una urgencia matemática. La naturaleza lógica de la filosofía exige una traslación del discurso poético hacia la dianoia; es decir, pensamiento que encadena, "logos sometido a ley" (Badiou, 2009, 63). En conclusión, cuando la poesía para Mallarmé se fundamenta en una ética del misterio, Badiou advierte que la filosofía existe para querer desgarrar ese velo. ${ }^{6}$

Como ejemplo, Badiou afirma: "Hoy, el poema está en el núcleo de la disposición filosófica, y el matema se encuentra excluido de ella. Es la matemática, la de nuestros días, la que [...] está en situación de exilio y de abandono a los ojos del filósofo" (Badiou, 2012, 156). De esta manera, para dar un paso más allá de la "edad de los poetas", es imperativo un discurso que pueda enlazar las "dos culturas". En esto se juega - según Badiou- el futuro de la filosofía.

\section{De la edad de las matemáticas}

Y es en este punto de extrema tensión del pensamiento donde somos convocados por la matemática. El imperativo es forjar

6 Para ello, ver el siguiente pasaje de Condiciones: “Cuando Parménides sitúa su poema en la invocación a la diosa, y comienza por la imagen de una cabalgata iniciática, creo que hay que decir que esto no es, que esto no es aún, filosofía. Porque toda verdad que acepte su dependencia del relato y de la revelación está todavía detenida en el misterio, en el cual la filosofía sólo existe para desgarrar ese velo" (Badiou, 2012, 84). 
un nuevo modo de intrincación de la matemática y de la filosofía, modo a través del cual se va a agotar el gesto romántico que aún nos rige.

Alain Badiou $(2012,158)$

Ahora bien, la importancia del matema en su pensamiento responde esencialmente a tres aspectos. En primer lugar, una razón platónica del lugar que ocupan las matemáticas en el orden del conocimiento metafísico; en segundo lugar, como ordenanza y sistematización del pensamiento en general, es decir: como dianoia; y finalmente, como acontecimiento matemático y por tanto cuerpo de lo verdadero.

Sobre la influencia platónica es importante señalar que Badiou encuentra en las matemáticas la posibilidad de Verdades Eternas. Las palabras de Aristóteles en la Física sobre "la inconmensurabilidad de la diagonal en relación al lado" es argumento suficiente para un nuevo gesto platónico. ${ }^{7}$ Los "gendarmes espirituales de Apolo" (Tasic, 2001, 10) creyeron que los enunciados matemáticos serían por siempre incorruptibles, eternos e inmutables.

Sin embargo, el fin del siglo xIx y principios del xx encuentran al pensamiento matemático en plena transformación hacia una etapa posmoderna. Allí, lo que se expresa es un conflicto entre la pureza heliotrópica de la antigüedad matemática y la crisis moderna de sus fundamentos. En el caso de Badiou, cabe señalar que el influjo matemático en su pensamiento platónico responde a las obras de Brouwer, Hilber y Cohen. Sin embargo, serán las figuras de Georg Cantor y Kurt Gödel quienes mayor impacto tendrán en su concepto de acontecimiento matemático. En efecto, el transfinito y el teorema de incompletitud constituyen para el francés una operación 'de-suturadora' de la condi-

7 Nos referimos al siguiente pasaje de la Física de Aristóteles: “Es claro entonces que todo no-ser no está en el tiempo, por ejemplo, las cosas que no pueden ser de otro modo (que como no-ser), así por ejemplo la conmensurabilidad de la diagonal en relación con el lado" (Aristóteles, 2008, 281). 
ción del matema. De esta manera, la filosofía de la matemática se eleva frente a las demás disciplinas porque comienza a haber un cuestionamiento sobre los conceptos de verdad, razón y lenguaje, inagurando de esta manera un giro metafísico y posmoderno.

Por tanto, es a partir de esta crisis de los fundamentos en pleno siglo xx que Badiou apunta a la matemática como fuente de acontecimiento y por tanto casa del ser. El filósofo señala dos direcciones: por un lado, la matemática se revela como una ciencia de hondura filosófica y por otro, como código del 'ser-en-sí y por tanto como lenguaje ontológico. Porque sólo la matemática puede expresar la presentación de la presentación: "Las matemáticas son más exactamente el único discurso que 'sabe' absolutamente de qué habla" (Badiou, 2003, 17). Por tanto, y dada su capacidad de abstracción frente a la materia, la matemática se convierte en el corazón de la condición científica y propulsora de un metalenguaje capaz de inscribir una tensión ontológica.

Ahora bien, examinaremos brevemente los tres conceptos nodales de la ontología matemática que propone el francés: lo Uno, lo múltiple y el vacío. Cabe señalar que, a diferencia de Heidegger, el ser se mantiene al margen de toda presentación. "Lo sustantivo no es el retiro del ser sino su renuncia a ser presentado como tal" (García Ponzo, 2011, 58). En definitiva, mientras que en la ontología poética el ser es presentado (como sucis: ser-ahí-manifestándose), en el matema hay una sustracción de esa presencia. En otras palabras, el matema no se detiene en el acontecimiento particular, sino que analiza el recorrido de su verdad.

Para ello es necesario retomar algunos conceptos de Cantor y la teoría de conjuntos, espcíficamente el singular problema de lo Uno. Como podemos deducir, todo conjunto matemático está compuesto por elementos; sin embargo, cada elemento se conforma a sí mismo como un conjunto, de tal manera que dentro de cada elemento hay al menos un par de elementos nuevos. Podemos intuir con Badiou que en las matemáticas de Cantor no 
hay posibilidad de lo uno, puesto que dentro de lo uno convive lo múltiple.

Badiou extiende esta sustracción a toda la filosofía. En consecuencia: lo 'uno-no-es'. Esto significa, por un lado, romper con todo filosofema de lo uno, y por otro, coronar el concepto de lo múltiple. Empero, no podemos asegurar que el ser sea lo múltiple, sino que esencialmente se configura en lo múltiple. "En efectos tales quiere decir: aquello que es presentado es la situación ontológica es lo múltiple, sin otro predicado que su multiplicidad. La ontología, en tanto exista, será necesariamente ciencia de lo múltiple en tanto múltiple" (Badiou, 2003, 38).

Cabe señalar que, contrario a lo que uno podría pensar en su afirmación, el ser no es matemático. Badiou no cae en el pitagorismo ontológico, sino que comprende a las matemáticas como el único lenguaje capaz de sustraer el ser. De esta manera se puede afirmar que las matemáticas son una suerte de metaontología: no revela el ser, sino que presenta las condiciones para sustraerlo.

De esta manera, el ser (como conjunto) exige lo múltiple. Cerrar el ser, como afirma la segunda ley de la termodinámica, supondría su muerte. ${ }^{8}$ Por esta razón, Badiou visualiza un ser abierto, porque es su cambio, su transformación, donde se proyecta lo verdadero. En este sentido, las verdades son producto de la multiplicidad múltiple, es decir: el elemento nuevo que viene a reconfigurar el ser como conjunto. Ese elemento, de sitio $\mathrm{x}$ (conjunto o condición, como lo llama Badiou) es necesariamente aquello que mantiene vivo el sistema, puesto que permite su movimiento. Este fenómeno, este elemento, será esencialmente su vacío: "El vacío nombraría la causa material de la traslación" (Badiou, 2003, 88).

8 Con respecto a este punto, Ilya Prigogine argumenta: “Esta distinción se halla contenida en la formulación de la segunda ley, que postula la existencia de una función, la entropía (entropía, en griego, significa evolución), que, en un sistema aislado, sólo puede aumentar debido a la presencia de procesos irreversibles, mientras que se mantiene constante durante los procesos reversibles. Por lo tanto, en un sistema aislado, la entropía alcanza al final un valor máximo cuando el sistema llega al equilibrio y cesa el proceso irreversible" (Prigogine, 1993, 18). 
Como podemos inferir de las palabras del filósofo, el vacío es el operador para el cambio del ser. Es, por lo pronto, potencia de lo verdadero. Y ontológicamente, si afirmábamos que el ser se presenta como múltiple, el ser-en-tanto-ser es aquello que necesariamente empuja la condición hacia lo abierto. Por ende: "el vacío es el nombre del ser" (Badiou, 2003, 71).

Por otro lado, la traslación que para los atomistas toma la forma de un mar vacío, adquiere aquí un movimiento inverso. El vacío no es, en efecto, el espacio general del movimiento, sino más bien el elemento de todo conjunto. Incluso, podríamos afirmar que el vacío es el elemento de todo conjunto y por tanto llave del ser. Paradójicamente, sin vacío habría más bien la nada.

La noción del vacío en Badiou se asemeja al concepto griego de ápeiron (forma de lo indefinido e ilimitado) que encuentra en las matemáticas transfinitas su actualidad en el campo de la filosofía matemática. El problema del infinito (en su estadio contemporáneo) ocupa para las matemáticas el espacio fundamental de ese vacío. Al igual que el infinito es una potencia para Aristóteles, el vacío se constituye, por tanto, como la potencia creadora de cualquier ontología posible. Por tanto, a partir de la identificación del infinito con el vacío, Badiou señala los descubrimientos matemáticos de Dederkind, Cantor y Gödel como acontecimientos ya sea por su exploración del infinito como por expandir los límites de las nociones de lenguaje y verdad en el campo de la matemática, potenciando de esta manera el pensamiento ontológico.

Es evidente, de este modo, el lugar central que ocupan las matemáticas de Dederkind, Cantor y Gödel no tan sólo como acontecimiento, sino como puesta en abismo de la propia condición del matema. Por tanto, ese espacio proyectado por el infinito es, a efectos, el símil matemático del vacío ontológico.

\section{Conclusiones}

De forma conclusiva, en la búsqueda por superar el ostracismo científico de los románticos, el filósofo retoma el gesto plató- 
nico contra la sofística para proyectar, de esta manera, el fin de la "edad de los poetas". Lo que el filósofo pretende es terminar con el precepto posmoderno de que la verdad no existe, es inútil y con ella toda metafísica y ontología se ven aniquiladas. Para Badiou volver a la filosofía moderna requiere imperativamente retomar el concepto de verdad como centro gravitatorio de toda metafísica posible.

Resulta imperioso, en este sentido, el cambio de paradigma que va del objeto poético al matemático. La voluntad por unificar ambos discursos se justifica bajo la necesidad técnica de la filosofía por expresar un lenguaje sometido a ley. Por otro lado, la traslación del acontecimiento poético al matemático ilumina ya no sólo las verdades que circulan en el pensamiento, sino que también permite la aproximación al lenguaje ontológico. En ambos casos el sentido está regido por la evolución de un vacío. La diferencia, en todo caso, se encuentra en su naturaleza: o bien como misterio de la presencia en el ejemplo poético de Heidegger, o bien como subconjunto de todo elemento que está incluido universalmente en el ejemplo matemático de Cantor.

Sin embargo, la plasticidad con la que Badiou maneja las teorías de la matemática y la poesía lo ha convertido en objeto de duras críticas. La academia norteamericana liderada por Alan Sokal y Fredric Jameson es escéptica sobre las capacidades técnicas del francés para abordar dichos estudios. En el ya famoso libro Imposturas intelectuales, Sokal y Bricmont acusan a Badiou de irresponsable a la hora de unificar el psicoanálisis lacaniano y la política con la teoría de conjuntos (Sokal y Bricmont, 1999, 179). Por otro lado, Jameson le dedica un férreo artículo que, entre otras cosas, atiende irónicamente la matematización de elementos estrictamente filosóficos (Jameson, 2017, 113).

En cambio, es importante señalar que Badiou no reniega de sus limitaciones, pero siempre acorazado en la gesta platónica de los universalistas, defendiendo un discurso transdisciplinario sobre la presencia de lo verdadero. En este sentido, las matemáticas transfinitas de Cantor le sirven para comprobar la imposibilidad 
de lo uno, ya sea tanto en la ontología como en la metafísica en general. Además, encuentra en la multiplicidad la cuenta matemática de la verdad. El nombre de esta invariación es, justamente, el acontecimiento. Pero para que lo múltiple sea, el contenido del 'ser-en-sí debe ser el vacío. Puesto que el vacío es la traslación que promueve lo múltiple y por tanto irrumpe contra lo uno.

Pero aún más, la matemática cumple un papel fundamental no sólo como discurso del ser, sino como soporte para el anudamiento de los saberes. En efecto, volver a unir matemática y poesía es la operación necesaria para quien quiera superar el fin de la filosofía. Por este motivo, Badiou no sólo se convierte en un emblema del pensamiento contemporáneo, sino también en uno de sus más importantes ontólogos, disciplina que creíamos suturada en su detención poética.

\section{Referencias bibliográficas}

Aristóteles (2008) Física, Madrid, Gredos.

Badiou, A. (2003) El ser y el acontecimiento, Buenos Aires, Manantial.

Badiou, A. (2007) Manifiesto por la filosofía, Buenos Aires, Nueva Visión.

Badiou, A. (2009) Pequeño manual de Inestética, Buenos Aires, Prometeo Libros.

Badiou, A. (2012) Condiciones, México, Siglo XXI.

García Ponzo, L. (2011) Badiou. Una introducción, Buenos Aires, Editorial Quadrata.

Habermas, J. (2012) El discurso filosófico de la modernidad, Buenos Aires, Katz.

Hersch, J. (2010) El gran asombro. La curiosidad como estímulo en la historia de la filosofía, Barcelona, Acantilado.

Jameson, F. (2017) "Badiou y la tradición francesa", The Left Review, 102, 100-119.

Muñoz Martínez, R. (2006) Tratamiento ontológico del silencio en Heidegger, Sevilla, Nueva Mínima del CIV. 
Prigogine, I. (1993) ¿Tan sólo una ilusión? Una exploración del caos al orden, Barcelona, Tusquets.

Rubín, A. (2016) Vivir el acontecimiento. Aproximaciones desde el pensamiento contemporáneo, Santiago de Compostela, Universidad de Santiago de Compostela.

Santiago, G. (2012), “Badiou: 'En filosofía es importante tener un adversario"', La Nación, 25 de mayo. Obtenido el 20/01/2019 en www.lanacion.com.ar/cultura/badiou-en-filo-sofia-es-importante-tener-un-adversario-nid1475204.

Snow, C. P. (2000) Las dos culturas, Buenos Aires, Nueva Visión.

SoKAL, A. y Bricmont, J. (1999) Imposturas intelectuales, Barcelona, Paidós.

TAsic, V. (2001) Una lectura matemática del pensamiento postmoderno, Buenos Aires, Ediciones Colihue. 
